\title{
Correlation of maternal anxiety and some of neonatal outcomes
}

\begin{abstract}
Maternal anxiety has different effects on pregnant women and their newborns. Several studies have examined the negative consequences of this disorder on the newborn. Aim of Our study was to determine the correlation between the anxiety maternal of third trimester and type of delivery, gestational age in delivery, newborn birthweight, newborn Apgar score, and neonatal pain. In this correlational and longitudinal study, 75 pairs pregnant women (in third trimester) and their newborns were selected through convenience sampling from Hospitals in Bandar Abbas, Iran in 2012. The data were collected using the Spielberger's Anxiety Inventory (STAI), a checklist for recording pregnancy outcomes (type of delivery, gestational age in delivery, newborn birthweight, newborn Apgar score), and neonatal Infant Pain Scale (NIPS). Finally, the data were analyzed using descriptive and analytical statistics (Pearson correlation, $T$ test) and SPSS software. The results showed that there was no significant relationship between state and trait pregnancy anxiety with variables such as type of delivery, gestational age in delivery, newborn birth weight, newborn Apgar score $(\mathrm{P}>0.05)$. But there was a reverse correlation between state and trait anxiety of mothers and neonatal pain, that was significant between the trait anxiety and the pain $(\mathrm{p}=0.13$; $\mathrm{r}=-0.176)(\mathrm{p}=0.04 ; \mathrm{r}=-0.238)$. According to lack of relationship between maternal anxiety and some neonatal outcomes, it is recommended that a study be conducted with a larger sample size. According to relationship between maternal anxiety and neonatal pain, it is possible that the cause of poor response to pain, weakening of the all of the behavioral symptom in newborn that are caused by stress transfer from pregnant mother to fetus, therefore, should not be overlooked for pain in the newborn and palliative cares. Therefore, prevention of maternal anxiety seems appropriate with screening methods.
\end{abstract}

Keywords: maternal anxiety, neonatal pain, neonatal outcome, risk factors
Volume 4 Issue 4 - 2018

\author{
Shilan Azizi,' Narges Sadeghi² \\ 'Ph.D Student in Nursing, Department of Nursing, Bandarabbas \\ Branch, Islamic Azad University, Bandarabbas, Iran \\ ${ }^{2} \mathrm{PhD}$ in nursing, Community Health Research Center, Isfahan \\ (khorasgan) Branch, Islamic Azad University, Isfahan, Iran
}

Correspondence: Shilan Azizi, Department of Nursing, Bandarabbas Branch, Islamic Azad University, Bandarabbas, Iran, Email azizi.sh23@yahoo.com

Received: May 30, 2018 | Published: July 12, 2018

\section{Introduction}

Anxiety is one of the most common psychiatric disorders in pregnancy ${ }^{1}$ reported about $22 \%$ prevalence of anxiety in Swedish pregnant women, ${ }^{2}$ while It has been reported about $29.7 \%, 28.6 \%$, and $39.6 \%$ in the first, second, and third trimesters of pregnancy in Iran, respectively ${ }^{3}$ and in the third trimester it is more prevalent than the first six months of pregnancy. ${ }^{4}$ Anxiety during this period is associated with different effects on mother and fetus and eventually newborn. some studies have shown the negative effects of this anxiety on the newborn, including low birthweight, early birth, low apgar score, effects on neonatal mental health, fetal distress and intrauterine growth retardation, less power to respond to stimuli, Long-term hospitalization. The existing literature reported a strong correlation between the psychological health of the newborn and the mother. ${ }^{5}$ However, maternal, emotional and psychological states vary in different pregnancy stages; depression and fatigue are most common in the first trimester and some more positive emotions emerge in the second trimester. In the third trimester, the mother is depressed and anxious due to worrying about the delivery and whether the newborn is healthy or not. ${ }^{6}$ Thus, it is of great importance to study the health of newborns, and pay special attention to pregnancy and controlling anxiety disorders in pregnant women, for long-term physiological and emotional health. The aim of this study was to determine the correlation between the anxiety maternal of third trimester and type of delivery, gestational age in delivery, newborn birthweight, newborn Apgar score, and neonatal pain.

\section{Materials and methods}

This study was a correlational and longitudinal study in pregnant women in the third trimester of pregnancy and their newborns after birth. Sample volume was estimated about 75 by taking into account the confidence interval (0.95), the power $(0.8)$, and correlation coefficient $(0.238)$ of previous studies. However, due to samples loss, forasmuch as that all women did not enter the study simultaneously, and the long sample selection process ( 4.5 months), the probable rate of sample loss in the 1st month after the beginning of the study was calculated as $30 \%$. Thus, 110 pregnant women were selected with convenience sampling from the three hospitals of the five hospital of Bandar Abbas city from Iran, Which had newborn, delivery, and women's units (only three hospitals issued permission for research). The sampling process was continued from July until December 2012. Finally, 75 pairs of women and newborns remained until the end of the study. The main reasons for sample loss were the following: lack of willingness to participate and collaborate; emigration; delivery in hospitals outside of Bandar Abbas city; delivery in other provinces; intrauterine fetal death; and early delivery. The instrument for collecting data in this study was the Spilberger Anxiety Inventory (STAI), a checklist for recording pregnancy outcomes (type of delivery, gestational age in delivery, newborn birthweight, newborn Apgar score), and the NIPS for measuring the pain in response to postnatal vaccination. Inclusion criteria included single pregnancy, being Iranian, having a gestational age 29 to 42 weeks, having no known disorder of mood or thinking, not taking anti-anxiety drugs and antidepressants. Exclusion criteria 
also included high risk pregnancy (diabetes, high blood pressure, placental dysfunction, and stillbirths), mothers who were not able to continue cooperation during the study for any reason (reluctance to continue cooperation, migration, death), neonates with congenital defects, receiving sedative drugs in the neonates was considered within 4 hours before the examination of the pain, sleepiness, and hyporeflexia of the newborn. Finally, the data were analyzed using descriptive and analytical statistics (Pearson correlation, T test) and SPSS software. Ethical considerations were observed.

\section{Findings}

The mean age of the mothers was $28.28 \pm 6.59$ years, the mean anxiety score was $41.48 \pm 10.45$ and mean trate anxiety score was $41.62 \pm 9.52$, the mean of the gestational age was $38.07 \pm 1.52$, The mean birthweight of the newborn was $3020.447 \pm 47.93$, the mean score of the first minute apgar was $8.66 \pm 0.64$, the fifth minute apgar was $9.83 \pm 0.42$ and the mean pain score was $5.85 \pm 1.84 .72 \%$ of mothers were multiparous. $24 \%$ had a history of abortion and $76 \%$ had no history of abortion. $40 \%$ had normal vaginal delivery and $60 \%$ had cesarean section delivery. The results showed that there was no relationship between type of anxiety (state and trate) with type of delivery, gestational age in delivery, newborn birthweight, newborn Apgar score of first minute and fifth minute $(\mathrm{P}>0.05)$. But there was an inverse relationship between the pain score of the newborn with state anxiety $(\mathrm{P}=0.13 ; \mathrm{r}=-0.176)$ and the pain score with trate anxiety $(\mathrm{P}=0.04 ; \mathrm{r}=-0.238)$.

\section{Discussion}

The results of the study showed that there is no relationship between the state and trate anxiety of pregnant women with type of delivery, gestational age in delivery, newborn birthweight, newborn Apgar score. But there is a reverse relationship between the state and trate anxiety of pregnant women with the score of the neonatal pain during vaccination. Although the correlation of 0.23 does not indicate a strong relationship, it can be concluded that, based on the negativity of this correlation, in the correlation between the state anxiety and the trate anxiety with the pain of the newborn, this may be due to an increase in the amount of anxiety in pregnancy, decrease Sensitivity and behavioral response of the newborn to pain resulting from invasive procedures. The results of studies ${ }^{7-11}$ also showed that increasing the anxiety of pregnancy, by affecting on the mechanism of pain control, reduces the sensitivity of the infant to pain and then the neonatal behavioral responses to the painful stimulus are weakened ${ }^{7,8,10-12}$ Some studies not find any association between maternal anxiety and newborn birth weight. ${ }^{1,12}$ But Nasreen and Shayeghian ${ }^{5,13}$ had contradictory results, which may be due to differences in studied populations and study methods. In order to find out the relationship between the state and trate anxiety of pregnant women with the gestational age in delivery, our results are consistent with the study by Alipour. ${ }^{12}$ Shahhosseini ${ }^{14}$ contrary to the results of our study, reported that the anxiety of mothers in the third trimester of pregnancy would cause preterm labor. Completion time and different types of questionnaires for assessing anxiety in pregnancy and different cut-off scores in studies can be one of the reasons for the incompatibility of these findings with other research. The results of our study showed that there is no relation between the score mean of state and trate anxiety of pregnant women in the third trimester of pregnancy with the score mean of the first and fifth minute newborn Apgar score, which is consistent with the results of ${ }^{12,15}$ and with results of some studies is inconsistent ${ }^{1,16}$ also, there was no relationship between the mean of state and trate anxiety of pregnant women in the third trimester of pregnancy with the type of delivery, while $60 \%$ of mothers had cesarean section and $40 \%$ had normal vaginal delivery. According to the researcher, the probable cause of the difference was the difference in the time of anxiety measurement and the use of different tools for measuring anxiety. According to the results of other studies, maternal anxiety in the third trimester of pregnancy may be an independent risk factor for type of delivery and neonates negative outcomes such as preterm delivery, low birthweight and low Apgar score are not considered, but other factors with anxiety may be involved in the occurrence of these negative outcomes that have not been addressed in this study.

The present research has some limitations. The first one is that genetic impacts on stress regulation and fetal pain perception cannot be separated from direct effect of anxiety and stress during the pregnancy period on the fetus. The second is long time of sampling due to the time of limit on gestational age and location limitation on women referring to hospitals. The third is lack of measurement of emotional and social support from the family and community that could be involved in anxiety. The strengths of our study are longitudinal design and present research was conducted for the first time in Iran.

\section{Conclusion}

Considering the lack of relationship between maternal anxiety and some neonatal outcomes, it is suggested that a study be conducted with a larger sample size and regarding the relationship between anxiety and pain, even if there are no behavioral or physiological symptoms due to the lower ability of some neonates to respond to pain, pain should be considered, Because the cause of weak response to pain may be the weakening of all signs of behavioral in the newborn that is caused by stress transfer from the pregnant mother to the fetus. On the other hand, in view of the long-term complications of neonatal pain, anxious mothers should screen as soon as possible through pregnancy anxiety questionnaires so that their damaging effects on mother and their newborn can be prevented.

\section{Acknowledgments}

None.

\section{Conflict of interest}

The author declares that there is no conflict of interest.

\section{References}

1. Bödecs T, Horváth B, Szilágyi E, et al. Effects of depression, anxiety, selfesteem, and health behaviour on neonatal outcomes in a population-based Hungarian sample. Eur J Obstet Gynecol Reprod Biol. 2011;154(1):45-50.

2. Claesson IM, Josefsson A, Sydsjö G. Prevalence of anxiety and depressive symptoms among obese pregnant and postpartum women: an intervention study. BMC Public Health. 2010;10:766

3. Foruzandeh N, Delaram M, Deris F. Mental health status and its determinant factors in women who referred to health centers in Shahrekord 2001-2002. Journal of Reproduction \& Infertility. 2003;4(2):146-155.

4. Lee AM, Lam SK, Sze Mun Lau SM, et al. Prevalence, course, and risk factors for antenatal anxiety and depression. Obstet Gynecol. 2007;110(5):1102-1112. 
5. Shayeghian Z, Rasolzadeh Tabatabaey SK ,Seddighi looye E. Effect of Maternal Anxiety during Third Trimester on Pregnancy Outcomes and Infants' Mental Health. J of Hayat. 2009;14(3):1.

6. Hyde JS, Else-Quest N. Half the human experience. Tehran, Iran: Agah Arjmand Publication; 2010.

7. Hernández-Martínez C, Arija V, Balaguer A, et al. Do the emotional states of pregnant women affect neonatal behaviour? Early Hum Dev. 2008;84(11):745-750.

8. Oberlander TF, Eckstein Grunau R, Fitzgerald C, et al. Prolonged prenatal psychotropic medication exposure alters neonatal acute pain response. Pediatr Res. 2002;51(4):443-453.

9. France CR, Taddio A, Shah VS, et al. Maternal family history of hypertension attenuates neonatal pain response. PAIN®, 2009;142(3):189_193.

10. Davis EP, Glynn LM, Waffarn F, et al. Prenatal maternal stress programs infant stress regulation. J Child Psychol Psychiatry. 2011;52(2):119-129.
11. Field T, Diego M, Hernandez-Reif M, et al. Pregnancy anxiety and comorbid depression and anger: effects on the fetus and neonate. Depress Anxiety. 2003;17(3):140-151.

12. Alipour Z, Hajizadeh E, Lamyian M. Anxiety during pregnancy: a risk factor for neonatal physical outcome? The Journal of Urmia Nursing and Midwifery Faculty. 2011;9(1):30-38.

13. Nasreen HE, Kabir ZN, Forsell Y, et al. Prevalence and associated factors of depressive and anxiety symptoms during pregnancy: a population based study in rural Bangladesh. BMC Womens Health. 2011;11:22.

14. Shahhosseini Z, Abedian K, Azimi H. Role of anxiety during pregnancy in preterm delivery. Zums Journal. 2008;16(63):85-92.

15. Bazrafshan M, Rad AM. The effect of pregnant women's anxiety on apgar score and birth weight of newborns (2009). Scientific Journal of Hamadan Nursing \& Midwifery Faculty. 2009;17(12):58-68.

16. Misri S, Oberlander TF, Fairbrother N, et al. Relation between prenatal maternal mood and anxiety and neonatal health. Can J Psychiatry. 2004;49(10):684-689 